35 AN ULTRA-RAPID REVIEW APPROACH: AN INNOVATIVE SOLUTION TO MAKE EVIDENCE MORE RELEVANT FOR TIME-SENSITIVE POLICY-MAKING AND PROVIDING GUIDANCE FOR HEALTH SYSTEMS?

1,2,3 Lisa Affengruber, ${ }^{1,2}$ Gernot Wagner, ${ }^{1,2,4}$ Gerald Gartlehner. 'Department of Evidencebased Medicine and Clinical Epidemiology, Danube University Krems, Krems, Austria; ${ }^{2}$ Cochrane Austria, Danube University Krems, Krems, Austria; ${ }^{3}$ Department of Family Medicine, Care and Public Health Research Institute (CAPHRI), Maastricht University, Maastricht, Netherlands; ${ }^{4}$ RTI International, North Carolina, USA

\subsection{6/bmjebm-2019-EBMLive.43}

Objectives To make evidence relevant to the time-sensitive needs of decision-makers in policy and health care systems, rapid reviews have become a practical alternative to systematic reviews because of their fast provision of results. By streamlining methodological steps, the results of rapid reviews are available within a few weeks or months. Ultra-rapid reviews employ multiple methodological shortcuts, are conducted in a very short timeframe (a few days), and therefore provide evidence at the time needed. We tested the accuracy of an ultrarapid review approach that substantially reduced the effort for literature searches and study selection. Our approach combined an abbreviated literature search with single-reviewer literature screening. The aim of this methods project was to evaluate the sensitivity of an ultra-rapid review approach based on three case studies and to ascertain if it leads to different overall conclusions about beneficial and harmful treatment effects compared with a systematic review approach.

Method The ultra-rapid review approach consisted of strongly abbreviated literature searches and single screening of abstracts and full texts. As reference standards, we selected three ongoing Cochrane reviews that had not been published at the time of the methods study. Two Cochrane reviews addressed oncological topics (OT), one a public health topic (PHT). A search specialist conducted abbreviated literature searches consisting of a simplestructured Boolean search in combination with the similar articles function in PubMed. Authors of the three Cochrane reviews provided highly relevant, published studies as starter sets for the similar articles function in PubMed. Three reviewers screened records independently and in parallel. If they missed studies compared with the reference standard, we recalculated effect estimates and created new Summary of Findings tables. We surveyed the Cochrane review authors to solicit whether the detected bodies of evidence from the ultra-rapid reviews would change the conclusions of the Cochrane review.

Results The proportion of abstracts screened by ultra-rapid reviewers compared to the Cochrane reviewers ranged from $15 \%$ to $1 \%$. The ultra-rapid review approach identified $11 \%$ to $22 \%$ of the relevant publications (OT: 11\%-15\%; PHT: $13 \%-$ $22 \%$ ) and $38 \%-100 \%$ of the relevant studies (OT: 40\%-100\%; PHT: 38\%-48\%). For case study one (OT), the ultra-rapid review approach led to the same conclusions as the Cochrane review. For case study two (OT), the authors would have drawn the same conclusion for all three ultra-rapid reviews, but with less certainty. For case study three (PHT), the authors would have drawn the same conclusion for one ultra-rapid review, but with less certainty. For the other two ultra-rapid reviews, the authors assessed that a conclusion could not be drawn.

Conclusions For oncological topics, the conclusions of the ultra-rapid review approach changed less often compared to the public health topic. Based on these three case studies, an ultra-rapid review approach seems to be viable for narrow questions that can be answered with RCTs. However, to assess whether an ultra-rapid review approach could be an expedited solution for evidence-based decisions for policy or health care professionals further research with a larger sample size is necessary to confirm our findings.

\section{ASSESSING MEDICAL STUDENTS' COMPETENCY IN EVIDENCE-BASED MEDICINE USING THE ACE TOOL- A CROSS SECTIONAL STUDY OF MEDICAL STUDENTS ACROSS DIFFERENT STAGES OF THE CURRICULUM}

${ }^{1}$ Bharathy Kumaravel, ${ }^{2}$ Dragan Ilic, ${ }^{1}$ Claire Stocker, ${ }^{1}$ Peter Thomas. 'University of Buckingham Medical School, Buckingham, UK; ${ }^{2}$ Monash University, Melbourne, Australia

\subsection{6/bmjebm-2019-EBMLive.44}

Objectives The importance of teaching the skills and practice of Evidence-Based Medicine (EBM) for medical students has steadily grown in recent years. Alongside this growth is a need to evaluate the effectiveness of EBM curriculum as assessed by competency in the five 'A's': Asking, Acquiring, Appraising, Applying and Assessing (impact). A longitudinal, competency based, clinically integrated EBM theme, with assessments has been designed and implemented in the University of Buckingham Medical School (UBMS). The EBM curriculum is progressive with students taught to ask, acquire and appraise evidence in years one and two. In years three, students are asked to apply EBM in clinical practice and reflect on their experience. The aim of this study was to carry out a cross sectional study examining the feasibility of administering the 15-item Assessing Competency in EBM (ACE) tool and compare student performance in the ACE tool across different years of EBM training.

Method While initially testing the feasibility of administering the tool, we used paper-based assessment administered during the EBM teaching session. After successfully completing the feasibility phase, we administered the test through our virtual learning environment. Data was collected on student performances in the paper-based assessment for one cohort (third year students) and from the assessment in our online portal for first and second year students. Performance data in ACE was gathered from a cross-sectional sample of 212 medical students representing, first year, second year and third year cohorts. Total ACE scores, item discrimination and internal reliability were analysed.

Results Performance data from 212 students (83 first years, 83 second years and 46 third years) was compared via one-way ANOVA. No significant difference in means scores was observed across the years (mean scores 10.4, 10.22, 10.28). Individual item discrimination was good except for one item (item discrimination index ranging from 0.27-0.93), overall test reliability was 0.60 , with internal reliability consistent across most items (item total correlations were all positive ranging from 0.14-0.60).

Despite the ease of administering and scoring, the ACE tool may have a lower potential to discriminate between different levels of students' EBM competencies. The lack of correlation between test scores and levels of training maybe explained by the small sample size for third year students, and administration via paper-based test versus the online based test. The ACE uses dichotomous questions type, where even novice students could randomly guess answers and still score high.

Conclusions The ACE test was very easy to administer and score, compared to other validated EBM assessment tools, such 
as the Fresno. Students found it very useful as a learning resource, demonstrating the application of EBM steps of asking, acquiring, appraising and applying evidence to a realistic clinical scenario. It is feasible to administer the ACE tool as a formative assessment in undergraduate medical education. It is a valuable teaching tool to demonstrate the application of the first four steps of EBM to a clinical scenario. Further research is needed to compare feasibility and students' performances in assessment tools and suggest a taxonomy of tools to guide EBM educators.

\section{EVIDENCE-BASED MEDICINE COURSE AS PART OF AN INTERNATIONAL MEDICAL EDUCATION CURRICULUM IN A RUSSIAN MEDICAL SCHOOL}

${ }^{1}$ Ksenia Ershova, ${ }^{2}$ Sergey Astrakov, ${ }^{1}$ Vladimir Zelman, ${ }^{2}$ Elena Neporada, ${ }^{1}$ Holly Muir, ${ }^{1}$ Philip Lumb. 'Department of Anesthesiology, Keck School of Medicine at the University of Southern California, Los Angeles, USA; '2Department of Anesthesiology and Intensive Care, Vladimir Zelman School of Medicine and Psychology at the Novosibirsk State University, Novosibirsk, Russian Federation

\subsection{6/bmjebm-2019-EBMLive.45}

Objectives Russian medical schools are currently incorporating evidence-based medicine (EBM) principles into their curricula. However, many medical schools still lack ancillary courses on research methods, epidemiology, biostatistics, etc., that result in reportedly poor quality of medical research in Russia. ${ }^{1} 2$ Many reasons for the difficulties have been identified, including linguistic barriers, lack of expertise, limited international collaboration, and inadequate allocation of resources. We hypothesized that the addition of an elective EBM curriculum to students in a Russian medical school would develop skills in searching, interpreting and evaluating medical literature, essential components of EBM.

As part of an international collaborative education program between two departments of anesthesiology, the EBM elective curriculum was created and introduced to all medical students (years 1-6). We then assessed the value of the course, students' attitude towards EBM, the extent to which they are engaged with evidence-based methodology, and the ability to incorporate EBM principles into future practice.

Method The collaborative education program was created between the Department of Anesthesiology, Keck School of Medicine at the University of Southern California (KSOM, USA) and the Department of Anesthesiology, School of Medicine and Psychology at the Novosibirsk State University (NSU, Russia). The EBM course included 18 seminars and journal clubs on biostatistics, clinical epidemiology, research methodology and other aspects of EBM. Classes were transmitted through video chat from KSOM to NSU, were voluntary and held in English. The course evaluation took place at the conclusion of the first year and consisted of two questionnaires. The first (Qs\#1) was for the course participants and a second (Qs\#2) was generally distributed among all students to learn about the potential course benefits and their understanding of EBM principles and importance. The questionnaires were distributed online as a GoogleForm (all questions were mandatory, one response per IP) and responses were anonymous.

Results Twenty-five students participated in the course. From 152 responses on Qs\#1, only 76\% agreed that clinical decision-making should be based on scientific evidence. A median frequency of reading medical literature was reported as once a week in their native language and once a month in English. On a scale from 1 to 10 , respondents ranked their understanding of scientific manuscripts as $6.3 \pm 1.8$, their confidence in assessing the quality of research as $5.5 \pm 2.1$. Respondents rated their interest in staying current with literature as $9.0 \pm 1.5$, and a potential benefit from learning critical thinking as $8.8 \pm 1.6$.

In a set of matched questions in Qs\#2, course participants reported that the course was valuable, helped build confidence in assessing research articles, and improved critical thinking. Participants vs. non-participants are more familiar with EBM $(8.2 \pm 2.2$ vs. $6.6 \pm 2.8, \mathrm{p}$-value $=0.002)$ and read medical literature more frequently (native language $p$-value $=0.039$, English p-value $=0.003$ ).

Conclusions Based on prior publications, ${ }^{1}{ }^{2}$ some Russian medical schools lack an EBM curriculum. By surveying a general medical student population, we found a discrepancy between the current and desired level of understanding of EBM indicating an unmet need. Currently, students feel insecure in applying EBM principles and have a deficiency in reading medical literature, especially in English. The EBM course, one component of an international education program, was established to create a scientific-centered learning environment, to provide expertise in EBM, and to help students overcome linguistic barrier. The participant evaluations showed that the course addressed positively the demonstrated needs of students and improved their reading and understanding of medical literature. We present evidence that reflects need for and benefits of including EBM in the medical curriculum. These findings indicate that international collaboration enhanced the medical students' educational experience by augmenting previously unavailable curricular content and should be continued.

\section{REFERENCES}

1. Telen, Marilyn J. 2014. "Teaching Evidence-Based Medicine in the Former Soviet Union: Lessons Learned." Transactions of the American Clinical and Climatological Association 125: 88-102; discussion 102-3. https://www.ncbi.nlm.nih.gov/ pubmed/25125721.

2. Vlassov, Vasiliy V. 2017. "Russian Medicine: Trying to Catch up on Scientific Evidence and Human Values." The Lancet 390 (10102): 1619-20. https://doi.org/ 10.1016/S0140- 6736(17)32382-6.

\section{TEACHING EVIDENCE BASED PRACTICE - IT'S TIME TO TACKLE THE 'APPLY' DOMAIN}

${ }^{1}$ Eve O'Toole, ${ }^{2}$ Niamh O'Rourke. 'National Cancer Control Programme, Health Service Executive, Dublin, Ireland; ${ }^{2}$ Clinical Effectiveness Unit, Department of Health, Dublin, Ireland

\subsection{6/bmjebm-2019-EBMLive.46}

Objectives In 2017 Evidence Based Practice Ireland (EBPI) was established to promote the use of evidence-based practice in the healthcare system in Ireland with the ultimate goal of improving patient outcomes. One of the challenges to the widespread adoption of the EBP paradigm is the lack of teaching of EBP to healthcare professionals. The teaching taking place rarely addresses the domain of how to apply evidence in practice. To address this deficit a three-day workshop in EBP was held in Ireland in March 2019 for practicing healthcare professionals with an emphasis on putting evidence into practice. The aims of the workshop were to develop and deliver a teaching module on 'evidence into practice'. To empower healthcare professionals to become evidence-based practitioners. To build capacity in teaching EBP and to create international links by inviting speakers form the Centre for Evidence Based Medicine (CEBM) in Oxford. 